\title{
Angular subtense effects on perception of polar and parallel projections of cubes
}

\author{
ANDREA L. NICHOLLS \\ McMaster University, Hamilton, Ontario, Canada \\ and \\ JOHN M. KENNEDY \\ University of Toronto, Toronto, Ontario, Canada
}

\begin{abstract}
Many authors contend that the perception of 2-D drawings of a 3-D object is governed by polar projective geometry. A problem for this position is that observers accept parallel projections, which are not produced with polar projective geometry, as accurate representations of 3-D objects. In Experiments 1 and 2, we used two different standards of comparison to study the perceptions of three line drawings of cubes-correct polar projections of cubes with subtenses of $15^{\circ}$ and $35^{\circ}$, and a parallel projection-at five different angular subtenses. In Experiment 1, 14 observers judged each drawing when it subtended about $35^{\circ}, 15^{\circ}, 5^{\circ}, 4^{\circ}$, and $2^{\circ}$ in width. Subjects used an 8-point rating scale to compare each drawing with a correct polar projection of a cube subtending $35^{\circ}$, viewed with the drawing subtending $15^{\circ}$. As predicted, both polar projections had their highest ratings at their correct vantage points. Ratings for the parallel projection were highest at small angular subtenses and decreased when it subtended $35^{\circ}$. These findings were supported by a second experiment in which the $15^{\circ}$ polar projection was set at a $5^{\circ}$ viewing angle as a standard. In Experiment 3, 15 observers compared the three drawings, viewed at a second set of angular subtenses $\left(30^{\circ}, 35^{\circ}, 40^{\circ}, 45^{\circ}\right.$, and $50^{\circ}$ ), with a standard, the $35^{\circ}$ polar set at $45^{\circ}$. Ratings fell with increases in viewing angle, and the parallel projection was rated lowest. The results indicate that parallel projections are assessed as polar projections that are correct for objects at a small angular subtense. Furthermore, projections at a small angular subtense are robust; that is, they are acceptable over a wide range of angular subtenses. We suggest that robustness can be explained by the modest variability in the proportions of pictures of cubes subtending small angles.
\end{abstract}

An important problem for the study of the perception of pictures lies in explaining how accurate impressions of three-dimensional (3-D) scenes can occur through the viewing of 2-D pictures. Here, we will examine two projection systems that have been used extensively to create 2-D pictures-polar projection and parallel projection. In polar projection drawings, lines representing edges that recede from the viewer converge on the picture surface and, if extended, would meet at vanishing points. In parallel projection drawings, lines representing parallel, receding edges are drawn as parallel on the page.

Objects project light to a vantage point, creating an optic array at the point, in what is called natural perspective.

This work was supported by a grant from the National Science and Engineering Research Committee, Ottawa. We thank E. Lupo for her assistance with data collection, and P. Bennett, T. Flagg, G. Moraglia, G. K. Humphrey, and H. Sedgwick for valuable comments. A. L. Nicholls's present address is at the Schnurmacher Institute for Vision Research, SUNY/State College of Optometry, 100 East 24th Street, New York, NY 10010 . J. M. Kennedy's address is at the Department of Psychology, University of Toronto/Scarborough College, 1265 Military Trail, Scarborough, ON M1C 1A4, Canada.
In a traditional view of drawing systems, polar projection is described as producing a set of shapes, or artificial perspective, when a picture surface intersects the natural light array projected from the objects to the vantage point (Pirenne, 1970; White, 1967). The center of projection in artificial perspective corresponds to the apex of the visual angles in the eye. If a drawing is produced with the use of polar projection, at this vantage point the optic array from the drawing will geometrically match the optic array from the objects in the 3-D scene. Thus, a polar projection drawing, viewed from its correct vantage points, should provide the eye with accurate information about 3-D objects or scenes.

In contrast, the system of parallel projection does not require the use of a single, correct vantage point. Hence, parallel projection drawings do not offer the observer the same optic array as do the objects that they depict. However, parallel projection drawings are widely advocated as accurate and visually acceptable representations of 3D objects (Arnheim, 1974; Dubery \& Willats, 1983; Hagen, 1985, 1986). This raises problems for theories of the perception of pictures based on projective geometry, if only polar projections can provide the eye with a geometrical match to the optic array received from an object. 
Hagen $(1985,1986)$ provides an interesting resolution, arguing that both polar and parallel projection are present in natural perspective and are therefore available for use in artificial perspective. Parallel projection is said to be present for substantial objects that are located far away from the vantage point: they subtend such small angles at the vantage point that the light sent to the vantage point is effectively parallel. Parallel projection drawings may be polar projection drawings for objects subtending very small angles. However, parallel drawings are viewed at a wide range of angular subtenses, not just tiny ones. Here we will try to explain how this could be, in terms that fit Hagen's thesis.

To be truly parallel, light rays would have to come from an object at an infinite distance. However, Hagen (1985) suggests that a vantage point "assumed at a distance of 40 times the size of the object is effectively at optical infinity" (p. 60). That is, optical infinity is deemed to be present when the object subtends around $1.4^{\circ}$ of visual angle. Hagen's rule for optical infinity is derived from a comparison of the angular subtense of the front edge of a transparent one-unit 3-D cube, viewed at eye level, with the angular subtense of the rear edge of the cube, at different distances from the eye. At 40 units, the difference in angular size of the two edges is only $2.5 \%$ (Hagen, 1985). Hagen's notion is that, in principle, parallel projection drawings are an extreme example of the system of polar projection. But do they operate in vision as polar projections do, in practice? If so, they should have correct and incorrect vantage points. Notice that a picture can be viewed from two kinds of incorrect vantage points, yielding two types of geometrical transformations: magnification/minification, which results from displacement of the observer's vantage point along a perpendicular to the picture plane; and a second type, which results from a lateral displacement of the vantage point parallel to the picture plane (Cutting, 1987; Farber \& Rosinski, 1978; Rosinski \& Farber, 1980). For the moment, our interests lie in considering the effects of changes along the perpendicular to the picture plane.

The transformation resulting from perpendicular displacement is termed magnification when the vantage point is shifted closer to the picture plane than the projectively correct vantage point, and minification when the vantage point is moved farther away. The predicted perceptual effects of the magnification/minification transformation include distortions of interobject distance, texture density, relative size, shape, and orientation of the depicted layout (Cutting, 1986; Farber \& Rosinski, 1978; Lumsden, 1980; Rosinski \& Farber, 1980; Sedgwick, 1991).

In picture perception research, there is considerable debate about the extent to which perception follows the rules of polar projective geometry during change of vantage point and angular subtense (Cutting, 1986; Hagen, 1980; Kubovy, 1986; Pirenne, 1970; Rosinski \& Farber, 1980; Sedgwick, 1980). In some cases, authors find that perception is essentially compatible with projective geome- try and that the distortions predicted by the transformed virtual space are indeed perceived (Bengston, Stergios, Ward, \& Jester, 1980; Busey, Brady, \& Cutting, 1990; McGreevy \& Ellis, 1986; Rosinski, Mulholland, Degelman, \& Farber, 1980; Smith \& Gruber, 1958).

However, other evidence suggests that perception does not always conform to the geometry of polar projection (Busey et al., 1990; Farber \& Rosinski, 1978; Goldstein, 1987; Rosinski et al., 1980; Smith, 1958; Wallach \& Marshall, 1986). Since the discovery and documentation of the geometric rules for producing polar projection drawings in the Renaissance, many have noticed that for some polar projection drawings, departures from the correct vantage point do not have serious consequences for the drawings' visual impression (Edgerton, 1975; Kubovy, 1986; Pirenne, 1970; Veltman, 1987; White, 1967). Artificial perspective specifies one optically correct location for viewing a picture, and many theorists would agree that a polar projection viewed from this correct location appears to the observer as a faithful depiction of the original referent. Yet in many instances the picture is also said to be robust. It is an acceptable representation from many different vantage points (Arnheim, 1977; Cutting, 1987; Kubovy, 1986; Pirenne, 1970).

Consider the common experience of viewing photographs. It is likely that the casual observer does not view the photograph from the projectively correct perpendicular viewing distance for the magnification or minification factor. (Also, the viewer may not hold the picture at the correct angle.) Yet to the observer, the image does not appear noticeably distorted. It is robust. Only sometimes does the observer notice distortions, as when the picture is viewed at a much closer distance than the projectively correct viewing distance (or at a very oblique angle).

The fact that some polar projection drawings are robust creates problems for theories of picture perception based on projective geometry, since polar projective geometry predicts distortions for viewing from incorrect vantage points. Furthermore, the effects of vantage point change on the perception of parallel projection drawings needs to be tested. If Hagen's thesis is correct, it is important to ask whether parallel projection drawings are robust, and if so, why.

In this paper, we will test the robustness and fragility of a parallel projection drawing in comparison with polar projection drawings. The results will provide a basis for explaining both the robust and the fragile pictures.

We present three experiments in which we examined the perceptual effects of viewing polar and parallel projection drawings at a range of incorrect vantage points along a normal to the picture surface. Ratings on an 8-point scale relative to a standard of comparison were used to gauge the perceptual changes. If the parallel projection drawing is taken to be a polar projection drawing subtending a very small angle at its correct vantage point, its ratings relative to the standard should decrease at large angular subtenses. Each polar projection should be rated 
as its best at its correct vantage point. The range over which each drawing is rated highly should provide an indication of its robustness.

\section{EXPERIMENT 1}

\section{Method}

Subjects. Fourteen undergraduates, 9 females and 5 males, participated as part of an introductory sensation and perception course at Scarborough College. All had normal or corrected-to-normal vision.

Apparatus. The participants were seated in front of a screen (43 cm high $\times 69 \mathrm{~cm}$ wide) containing two peepholes (each $2 \mathrm{~cm}$ high $\times 2 \mathrm{~cm}$ wide) $31 \mathrm{~cm}$ apart. The center of each peephole was $26 \mathrm{~cm}$ from the table top.

Stimuli. Three line drawings of cubes (Figure 1) were displayed one at a time, set vertically behind the screen. The cubes were drawn as though a vertex and a convex corner faced the viewer, with the left and right faces placed symmetrically. One drawing was a parallel projection, $13.7 \mathrm{~cm}$ high $\times 12.7 \mathrm{~cm}$ wide. It subtended $15^{\circ}$ in width ( $16^{\circ}$ in height) at a distance of $48 \mathrm{~cm}$ from the peephole. Another was a polar projection drawing, with a moderate convergence of the lines showing the receding sides $(12.3 \mathrm{~cm}$ high $\times$ $12.3 \mathrm{~cm}$ wide). It subtended an angle of $15^{\circ}$ at its correct viewing distance of $48 \mathrm{~cm}$. A third drawing was a polar projection drawing with strongly convergent lines showing the receding sides. It was $11.8 \mathrm{~cm}$ high $\times 13 \mathrm{~cm}$ wide and subtended $36^{\circ}$ in width and $33^{\circ}$ in height at its correct viewing distance of $20 \mathrm{~cm}$. For convenience, we will describe it as the $35^{\circ}$ drawing. Each drawing was done with black lines, $2 \mathrm{~mm}$ in thickness, on a white background.

The three drawings described above were shown at five angular subtenses (i.e., five distances) with a standard of comparison that always remained at $15^{\circ}(48 \mathrm{~cm})$. The standard of comparison was the same as the strongly convergent drawing - a correct projection at $35^{\circ}(20 \mathrm{~cm})$-but placed at an incorrect viewing distance. The standard of comparison was always viewed through the left peephole. Placing the standard at an incorrect angular subtense (view-
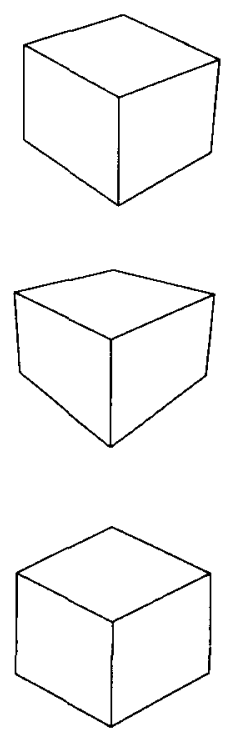

Figure 1. The moderately convergent polar projection (top), the strongly convergent polar projection (center), and the parallel projection (bottom).
Table 1

Mean Ratings for the Moderately Convergent Drawing, the Strongly Convergent Drawing, and the Parallel Projection Drawing at Five Distances (in Centimeters), Strongly Convergent Drawing as Standard (Experiment 1)

\begin{tabular}{ccccc}
$\begin{array}{c}\text { Angular } \\
\text { Subtense }\end{array}$ & Distance & $\begin{array}{c}\text { Moderately } \\
\text { Convergent }\end{array}$ & $\begin{array}{c}\text { Strongly } \\
\text { Convergent }\end{array}$ & $\begin{array}{c}\text { Parallel } \\
\text { Projection }\end{array}$ \\
\hline $35^{\circ}$ & 20 & 5.64 & 4.57 & 3.29 \\
$15^{\circ}$ & 48 & 7.21 & 2.36 & 5.43 \\
$5^{\circ}$ & 140 & 6.36 & 2.36 & 5.86 \\
$4^{\circ}$ & 200 & 6.36 & 2.64 & 5.50 \\
$2^{\circ}$ & 400 & 6.57 & 3.50 & 5.43 \\
\hline
\end{tabular}

ing distance) should have allowed it to appear imperfect, and to have opened the possibility that other drawings might appear to be "better." The drawings to be rated were viewed individually through the right peephole at angles of $35^{\circ}(20 \mathrm{~cm}), 15^{\circ}(48 \mathrm{~cm}), 5^{\circ}$ $(140 \mathrm{~cm}), 4^{\circ}(200 \mathrm{~cm})$, and $2^{\circ}(400 \mathrm{~cm})$. Appendix A contains exact measures of angular subtenses for the widths and heights of the three drawings at the five distances.

Procedure. The subjects were told to use the same eye when looking through both peepholes. First the subject looked through the left peephole at the standard of comparison for $10 \mathrm{sec}$. Then the subject had $10 \mathrm{sec}$ to look through the right peephole at the drawing to be rated. The subject judged which drawing was a better drawing of a cube. The subjects were instructed to compare the second drawing to the standard on an 8-point scale described as just barely better (5) or worse (4), a bit better (6) or worse (3), a fair amount better (7) or worse (2), or a great deal better (8) or worse (1). Each subject made 15 judgments in total, as each of the 3 drawings was shown at each of the 5 distances, in a randomized order counterbalanced across subjects.

\section{Results}

Table 1 shows clear distinctions between the mean ratings for the drawings. A 3 (projection type) $\times 5$ (viewing distance) repeated measures analysis of variance (ANOVA) performed on the data yielded significant main effects of projection type $[F(2,26)=27.13, p<.001]$ and distance $[F(4,52)=3.19, p<.05]$. A post hoc analysis of the main effect of projection type using a NewmanKeuls procedure showed that the mean ratings for the three projections were significantly different from each other. The overall mean ratings dropped significantly at $35^{\circ}$ $(20 \mathrm{~cm})$. However, these effects were qualified by an interaction between projection type and distance $[F(8,104)$ $=8.95, p<.001]$.

As Figure 2 shows, mean ratings for the parallel projection drawing remained stable from $15^{\circ}(48 \mathrm{~cm})$ to $2^{\circ}$ $(400 \mathrm{~cm})$, with a significant decrease to 3.29 at $35^{\circ}$ (Newman-Keuls post hoc analysis, $d f=52, p<.05$ ). Interestingly, means for the parallel projection drawing at $5^{\circ}(140 \mathrm{~cm}), 4^{\circ}(200 \mathrm{~cm})$, and $2^{\circ}(400 \mathrm{~cm})$ were not significantly different from means for the moderately convergent drawing at the same distances.

The moderately convergent drawing received a significantly higher mean rating of 7.21 at its correct viewing angle of $15^{\circ}(48 \mathrm{~cm})$ in comparison with the other four distances (Newman-Keuls, $d f=52, p<.05$ ), and its mean rating at $35^{\circ}(20 \mathrm{~cm}), 5.64$, was significantly lower than 


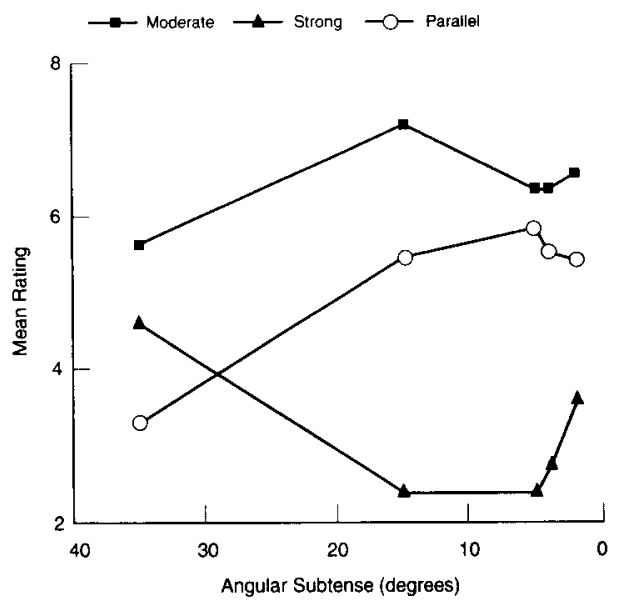

Figure 2. Strongly convergent drawing set at $15^{\circ}$ as standard: angular subtense versus mean ratings for Experiment 1.

the means for the other angles (Newman-Keuls, $d f=52$, $p<.05$ ). At all angles, its ratings were higher than those for the other two projections. Its mean ratings were significantly higher than those for strongly convergent drawing at all angles (Newman-Keuls, $d f=104, p<.05$ ).

Figure 2 shows that the mean rating for the strongly convergent drawing was significantly higher at its correct viewing angle of $35^{\circ}(20 \mathrm{~cm})$ than at other angles (NewmanKeuls, $d f=52, p<.05)$. Ratings decreased dramatically to 2.36 at $15^{\circ}(48 \mathrm{~cm})$ and $5^{\circ}(140 \mathrm{~cm})$. There was an unexpected increase in ratings from $4^{\circ}(200 \mathrm{~cm})$ to $2^{\circ}$ $(400 \mathrm{~cm})$ (Newman-Keuls, $d f=52, p<.05)$.

An interesting feature of the results was that only when the angular subtense was greatest was the mean for the strongly convergent drawing (4.57) higher than that for the parallel projection drawing (3.29).

A further comparison of interest is that between the mean ratings for the three projections at their correct vantage points (assuming that the parallel projection drawing is correct at $2^{\circ}$ ). Projective geometry predicts no differences between the ratings. Analysis shows that the mean rating for the moderately convergent drawing at $15^{\circ}(7.21)$ was significantly higher than the mean ratings for the strongly convergent drawing at $35^{\circ}(4.57)$ and the parallel projection drawing at $2^{\circ}(5.43)$.

\section{Discussion}

Perception of the drawings was affected by changes in angular subtense. Notably, there was a decrease in mean ratings for the parallel projection drawing when it subtended a large angle. Subjects reported that the parallel projection drawing looked distorted at $35^{\circ}$. The sides appear to diverge, rather than to remain parallel. Readers are invited to check this for themselves, by bringing Figure 1, the parallel projection drawing, close to the eye. The effect is pronounced and reliable.

For other vantage points, where the parallel projection drawing subtended small angles, the mean ratings remained consistent. The subjects seemed to find the paral- lel projection drawing equally acceptable for angles of $15^{\circ}$ and less. It seems likely that the range over which the parallel drawing is visually stable is much larger than $\mathrm{Ha}$ gen's $(1985,1986)$ calculations suggest.

Both the moderately convergent drawing and the strongly convergent drawing were rated most highly at their projectively correct distances. Ratings for the strongly convergent drawing decreased reliably with departures from its correct vantage point. The subjects reported that the strongly convergent drawing looked too sharp and too convergent when it was viewed with a small angular subtense. The moderately convergent drawing also shows a decrease in ratings away from its correct vantage point, but after this decrease, ratings remained remarkably stable over a wide range of subtenses. (Again the reader may wish to check this by using Figure 1.)

The subjects' ratings were not closely related to the ratio change of angle from the correct angle. The moderately convergent drawing changed by a factor of 7.5 from $15^{\circ}$ to $2^{\circ}$, and the strongly convergent drawing changed by a factor of 7 from $35^{\circ}$ to $5^{\circ}$. But the moderately convergent drawing was still rated highly at $2^{\circ}$, and the strongly convergent drawing's ratings plunged from 4.57 to 2.36 .

The moderately convergent drawing was highly robust. It was rated more highly than the standard from $2^{\circ}$ to $35^{\circ}$. The parallel projection drawing was also robust, but only from $2^{\circ}$ to $15^{\circ}$. The strongly convergent drawing was only deemed as good as the standard once, at its correct viewing distance. It appears to have been quite fragile, sensitive to any departure from correct viewing angle.

The strongly convergent drawing shows an unexpected increase in ratings-albeit a small one-at $2^{\circ}$, in comparison with $4^{\circ}$, contrary to the geometrical prediction of a steady decrease with increasing departures from the correct vantage point. This is anomalous. Furthermore, polar perspective geometry offers only a direct explanation of the individual trends: the higher rating for each of the convergent drawings at its correct vantage point, the decrease in ratings for the moderate convergent drawing when it subtended small angles, and the drop in mean ratings for the parallel projection drawing when it subtended a large angle. More indirect considerations will be needed to explain why ratings for one cube drawing were more robust than another.

One obvious explanation for some of the discrepancies from polar perspective geometry is that the strongly convergent drawing at $15^{\circ}$ was used as the standard and was always seen first. Although the subjects were told that the drawings could be rated as better or worse than the standard, they may have attached some special significance to the drawing used as the standard. We conducted the experiment again, using the moderately convergent drawing set at $5^{\circ}(140 \mathrm{~cm})$ as the standard. Notice that in Experiment 1 the strongly convergent drawing plunged in ratings from $35^{\circ}$ to $15^{\circ}$, whereas the moderately convergent drawing was barely affected by the change from $15^{\circ}$ to $5^{\circ}$. Accordingly, one should expect that the moderately convergent drawing set at $5^{\circ}$ would set a higher standard, 
suppressing and minimizing variability between the lower ratings but providing more room for variability in the higher ratings than in Experiment 1.

\section{EXPERIMENT 2}

\section{Method}

Subjects. Forty-five undergraduates, 41 females and 4 males, participated as part of a perception and cognition course at Scarborough College. All had normal or corrected-to-normal vision.

Apparatus and Stimuli. The three line drawings of cubes used in Experiment 1 were displayed at the same angles through the right peephole. However, the standard of comparison on the left side of the apparatus was the moderately convergent polar projection drawing, at $5^{\circ}(140 \mathrm{~cm})$

Procedure. The subjects were required to make their judgments as before.

\section{Results}

The mean ratings for the drawings at each distance are shown in Table 2 and Figure 3. A 3 (projection type) $\times$ 5 (viewing distance) repeated measures ANOVA yielded main effects of projection type $[F(2,88)=5.48, p<.001]$ and viewing angle $[F(4,176)=5.97, p<.01]$, as well as a projection type by viewing angle interaction $[F(8,352)$ $=7.91, p<.001]$. The moderately convergent drawing received a significantly higher mean at its correct angular subtense, $15^{\circ}$, than at other subtenses (Newman-Keuls, $d f=176, p<.05)$. The highest mean rating for the strongly convergent drawing, 4.09 , was at its correct subtense, $35^{\circ}$. The mean ratings for the moderately convergent drawing at $35^{\circ}$ and $15^{\circ}$ were not significantly different from each other, but they were significantly higher than the mean ratings at $5^{\circ}, 4^{\circ}$, and $2^{\circ}$ (Newman-Keuls, $d f=176, p<.05)$. The parallel projection drawing was rated lower than the moderately convergent drawing at all angular subtenses. The parallel projection drawing received its lowest mean rating, 2.73 , at $35^{\circ}$. However, post hoc analyses with the Newman-Keuls procedure showed that the mean ratings for the parallel projection drawing did not differ across the five angles. We attribute this to the fact that it was being compared with a higher standard than in Experiment 1, so that all its ratings were lower, and, as a result, differences were minimized.

Figure 3 shows an intersection of the curves for the parallel projection drawing and the strongly convergent draw-

Table 2

Mean Ratings for the Moderately Convergent Drawing, the Strongly Convergent Drawing, and the Parallel Projection Drawing at Five Distances (in Centimeters), Moderately Convergent Drawing as Standard (Experiment 2)

\begin{tabular}{ccccc}
\hline & & \multicolumn{3}{c}{ Drawing } \\
\cline { 4 - 5 } $\begin{array}{c}\text { Angular } \\
\text { Subtense }\end{array}$ & Distance & $\begin{array}{c}\text { Moderately } \\
\text { Convergent }\end{array}$ & $\begin{array}{c}\text { Strongly } \\
\text { Convergent }\end{array}$ & $\begin{array}{c}\text { Parallel } \\
\text { Projection }\end{array}$ \\
\hline $35^{\circ}$ & 20 & 4.87 & 4.09 & 2.73 \\
$15^{\circ}$ & 48 & 5.53 & 3.84 & 3.38 \\
$5^{\circ}$ & 140 & 4.96 & 2.60 & 3.36 \\
$4^{\circ}$ & 200 & 5.04 & 2.67 & 3.20 \\
$2^{\circ}$ & 400 & 4.76 & 2.38 & 3.42 \\
\hline
\end{tabular}

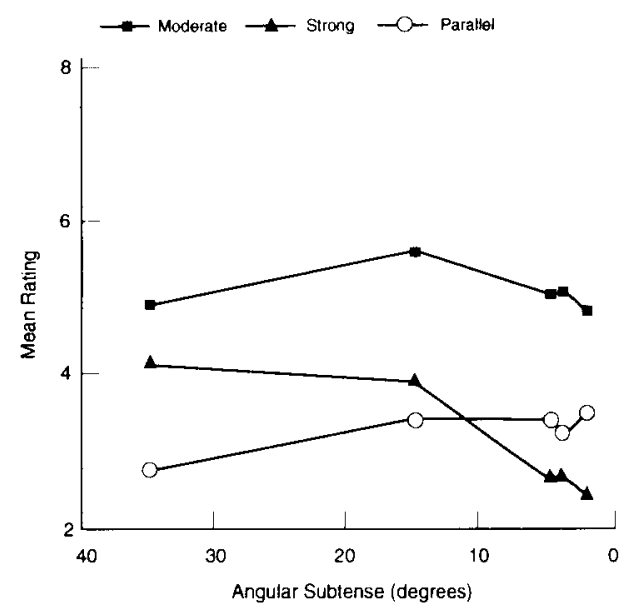

Figure 3. Moderately convergent drawing set at $5^{\circ}$ as standard: angular subtense versus mean ratings for Experiment 2.

ing between $15^{\circ}$ and $5^{\circ}$. The mean ratings for the strongly convergent drawing at $35^{\circ}$ and $15^{\circ}$ were significantly different from those for the parallel projection drawing across all angles (Newman-Keuls, $d f=352, p<.05$ ).

Analyses of the mean ratings for each projection type at its correct distance (assuming that the parallel projection drawing is correct at $2^{\circ}$ ) shows that the ratings for the moderately convergent drawing at $15^{\circ}$ were significantly higher than those for the strongly convergent drawing at $35^{\circ}$ (Newman-Keuls, $d f=88, p<.05$ ) and that the mean rating for the strongly convergent drawing was significantly higher than the mean rating for the parallel projection drawing at $2^{\circ}$ (Newman-Keuls, $d f=88, p<$ $.05)$. Ratings for the strongly convergent drawing did not increase at $2^{\circ}$. We suggest that the increase found in Experiment 1 is not a reliable finding.

\section{Discussion}

Ratings for the three different projections followed the predicted effects of polar projective geometry. Both polar projection drawings received their highest ratings at their correct vantage points. Ratings for the strongly convergent drawing decreased markedly with increased departure from its correct vantage point. As in Experiment 1, the ratings for the strongly convergent drawing and the moderately convergent drawing did not correspond to the ratios of the viewing angle to the correct subtense. The rating of the strongly convergent drawing at $5^{\circ}$ was 2.60 , whereas the rating of the moderately convergent drawing at $2^{\circ}$ was 4.76 , although both changed by a factor of 7 or 7.5 from their correct subtense. The parallel projection drawing was rated 3.42 at $2^{\circ}$, and remained stable, within a narrow range, to $15^{\circ}$ (falling no lower than 3.2). At $35^{\circ}$, it was rated lowest (2.73). The low rating was not significantly different from the ratings at other angles as a result of the use of a higher standard of comparison, we suggest. To check further on our analysis of the parallel projection cube drawing, we conducted a third experiment. 
The view that the parallel projection is perceived as a polar projection subtending very small angles predicts further decreases in ratings for angular subtenses larger than $35^{\circ}$. In Experiment 3, we tested this prediction. Following the same basic procedure, the subjects viewed the three drawings at angular subtenses of $30^{\circ}, 35^{\circ}, 40^{\circ}, 45^{\circ}$, and $50^{\circ}$. The standard of comparison was the strongly convergent drawing set at $45^{\circ}$.

\section{EXPERIMENT 3}

\section{Method}

Subjects. Fifteen undergraduates, 12 females and 3 males, participated to fulfill a research participation requirement for an introductory psychology course at Scarborough College. All had normal or corrected-to-normal vision.

Apparatus and Stimuli. The subjects viewed the three line drawings used in Experiments 1 and 2 through a peephole measuring $3 \times 3 \mathrm{~cm}$, centered in a screen measuring $34 \mathrm{~cm}$ wide and positioned $24 \mathrm{~cm}$ above the base of the screen, which was $42 \mathrm{~cm}$ high. A second similar peephole, in a similar screen, was located to the left. The right peephole was used for individually viewing the three projection drawings set at varying distances: $13 \mathrm{~cm}$, where the three drawings subtended $50^{\circ} ; 15 \mathrm{~cm}$ (or $45^{\circ}$ ); $17 \mathrm{~cm}$ (or $40^{\circ}$ ); $20 \mathrm{~cm}$ (or $35^{\circ}$, the correct angular subtense for the strongly convergent polar projection); and $25 \mathrm{~cm}\left(30^{\circ}\right)$. (More precise angular subtenses for the height and width of each drawing at the peephole are given in Appendix B.) The left peephole was used for viewing the standard of comparison, the moderately convergent polar projection set at $45^{\circ}(15 \mathrm{~cm})$.

Procedure. The subjects were required to look at the standard drawing through a peephole for $10 \mathrm{sec}$, after which they looked through a second peephole at one of the three drawings to be rated, set at one of the five distances, for $10 \mathrm{sec}$. The subjects used a rating scale as before.

\section{Results}

Table 3 shows mean ratings for each of the three projection drawings at each angular subtense (distance). A 3 (projection type) $\times 5$ (viewing distance) repeated measures ANOVA yielded main effects of projection type $[F(2,28)=34.77, p<.01]$ and viewing distance $[F(4,56)$ $=3.08, p<.05]$. As Figure 4 shows, the mean ratings for the parallel projection drawing were significantly lower than the means for the other two projection types (Newman-Keuls, $d f=28, p<.05$ ). For each of the three projection drawings, the mean ratings relative to the standard generally decreased with increases in angular sub-

Table 3

Mean Ratings for the Moderately Convergent Drawing, the Strongly Convergent Drawing, and the Parallel Projection Drawing at Five Distances (in Centimeters), Strongly Convergent Drawing as Standard (Experiment 3)

\begin{tabular}{ccccc}
\hline & & \multicolumn{3}{c}{ Drawing } \\
\cline { 3 - 5 } $\begin{array}{c}\text { Angular } \\
\text { Subtense }\end{array}$ & Distance & $\begin{array}{c}\text { Moderately } \\
\text { Convergent }\end{array}$ & $\begin{array}{c}\text { Strongly } \\
\text { Convergent }\end{array}$ & $\begin{array}{c}\text { Parallel } \\
\text { Projection }\end{array}$ \\
\hline $50^{\circ}$ & 13 & 4.47 & 4.47 & 2.27 \\
$45^{\circ}$ & 15 & 5.20 & 4.53 & 2.67 \\
$40^{\circ}$ & 17 & 5.60 & 5.33 & 2.73 \\
$35^{\circ}$ & 20 & 5.93 & 5.47 & 2.93 \\
$30^{\circ}$ & 23 & 6.27 & 5.20 & 2.93 \\
\hline
\end{tabular}

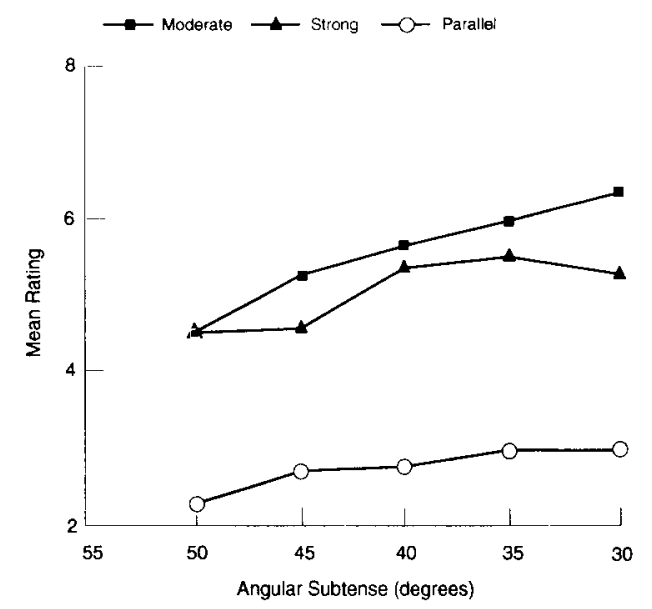

Figure 4. Strongly convergent drawing set at $45^{\circ}$ as standard: angular subtense versus mean ratings for Experiment 3.

tense (decreases in viewing distance). The overall ratings at $50^{\circ}$ were significantly lower than the ratings at the other four angular subtenses (Newman-Keuls, $d f=56, p<$ .05 ). The ratings at $30^{\circ}$ and $35^{\circ}$ did not differ significantly, but they were significantly higher than the ratings at $40^{\circ}$, $45^{\circ}$, and $50^{\circ}$ (Newman-Keuls, $d f=56, p<.05$ ). For the strongly convergent drawing, the highest mean rating was at $35^{\circ}$, its correct viewing angle.

\section{Discussion}

At large angular subtenses, the parallel projection drawing was rated significantly lower than either the strongly convergent drawing or the moderately convergent drawing. The strongly convergent drawing was rated most highly when closest to its correct viewing angle of $35^{\circ}$. Ratings decreased with increases in angular subtense. The moderately convergent drawing also showed decreases in mean ratings with increases in angular subtense. As in Experiments 1 and 2 , some of the findings are not consistent with polar projective geometry. Most notably, the moderately convergent drawing again received higher ratings than the strongly convergent drawing did, despite the fact that it was farther from its correct vantage point. Indeed, when the strongly convergent drawing was placed at its correct vantage point, the ratings were not significantly different from those for the moderately convergent drawing at the same distance. One possibility for the consistently high ratings for the moderately convergent drawing is that the effects of distortions resulting from departure from the correct vantage point are not perceptually salient. We will now turn our attention to a possible basis for this suggestion.

\section{GENERAL DISCUSSION}

Hagen $(1985,1986)$ suggested that parallel projection drawings are polar projection drawings for substantial objects subtending small angles. Our findings offer some sup- 


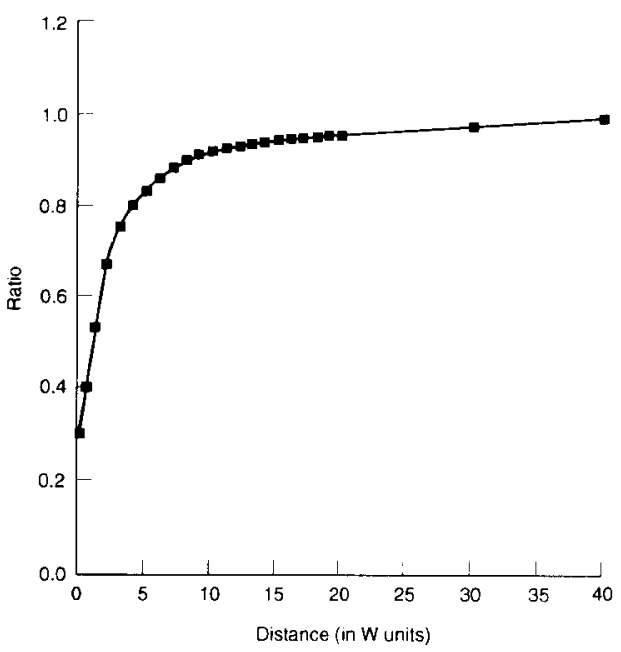

Figure 5. Distance versus angle subtended by the rear face of a 1-unit transparent 3-D cube as a proportion of the angle subtended by the front face.

port for this view. But we still need to explain the remarkable stability of some of the figures.

Ratings for the parallel projection drawing remain stable for a wide range of angular subtenses - from $15^{\circ}$ and lower-and do not begin to decrease until the subtense becomes quite large, possibly $35^{\circ}$. In Hagen's view, a visual angle of $1.4^{\circ}$ approximates optical infinity, but our findings suggest stability over much larger angles.

An explanation for perceptual stability may be found in the proportions of a projectively correct figure, and the variation in those proportions as a figure varies its distance and angular subtense from the correct vantage point.

Consider a transparent 3-D cube of width $w$. Let the observer's vantage point be placed in the middle of one of the cube's faces. That face will subtend $180^{\circ}$ at the vantage point. The rear face of the cube, distance $w$ away, will subtend about $53^{\circ}$. If the cube moves away from the vantage point by distance $w$, the front face will replace the rear face, and so must subtend $53^{\circ}$, while the rear face (now $2 w$ distant) will subtend about $28^{\circ}$. If the cube recedes by distance $w$ again, the rear face would subtend $19^{\circ}$. Successive retreats by distance $w$ will make the rear face subtend $14^{\circ}, 11.4^{\circ}, 9.5^{\circ}, 8.2^{\circ}, 7.2^{\circ}, 6.4^{\circ}, 5.7^{\circ}$, $4.8^{\circ}, 4.4^{\circ}, 4.1^{\circ}, 3.8^{\circ}, 3.6^{\circ}, \ldots$ The formula relating the angle subtended, $y$, to the distance, $d$, and the width, $w$, is $\tan (y / 2)=(w / 2) / d$.

A shift of distance $w$ produces sizable changes (as that from $53^{\circ}$ to $28^{\circ}$ ) when the cube is close and subtends a large angle (see Figure 5). The same change in distance results in quite minor angular changes (as that from $3.8^{\circ}$ to $3.9^{\circ}$ ) when the cube is farther away and subtends a smaller angle. The result is that any object with proportions like a cube's can change its distance a lot, while subtending a small angle, without producing a material effect on the angles that its parts subtend.
Let us see how this could affect visual criteria. It may be proportion-at-a-subtense, rather than just proportion or angle subtended, that vision weighs heavily.

The proportion $3.6^{\circ} / 3.8^{\circ}$ is 0.95 . The proportion $3.8^{\circ} / 4.1^{\circ}$ is $0.93 ; 4.1^{\circ} / 4.4^{\circ}$ is 0.93 ; and $4.4^{\circ} / 4.8^{\circ}$ is 0.92 . Therefore, a cube that is distant enough from the vantage point to have a small subtense can move a distance equal to several times its width before the projected proportions of the rear and front faces change appreciably. Compare a cube subtending a large angle. The proportion $53^{\circ} / 180^{\circ}$ is $0.29 ; 28^{\circ} / 53^{\circ}$ is $0.52 ; 19^{\circ} / 28^{\circ}$ is $0.68^{\circ}$; and $14^{\circ} / 19^{\circ}$ is 0.74 . Evidently, if a cube with a large subtense at the vantage point moves by its own width, the projected proportions of the rear and front face alter emphatically.

These indisputable facts of geometry have a clear role to play in vision's use of perspective, if vision uses the proportions that we have calculated. Although our calculations are based on a vantage point facing the center of the square face of a transparent 3-D cube, in principle the effects remain the same if the cube is opaque, and in another orientation, as that with a vertex facing the vantage point. Objects depicted with proportions that are appropriate for small subtended angles can be viewed at a large range of distances before they will look awry.

The application to pictures of cubes is straightforward. Consider someone inspecting a drawing of a cube with the rear subtending $3.6^{\circ}$ while the front is $3.8^{\circ}$. This is the 0.95 proportion. Assume that the observer appreciates that this is an appropriate rendering of the cube. Assume further that the observer finds that this drawing is still reasonably acceptable just to the distance when the front subtends $11.4^{\circ}$. At $11.4^{\circ}$, the correct proportions for the rear and front are $0.83\left(9.5^{\circ} / 11.4^{\circ}\right)$. The difference between the correct proportions and this limit of "reasonably acceptable" is $0.12(0.95-0.83)$. It seems that this observer tolerates deviations from the correct proportions of up to about $12 \%$. If so, the picture of the cube could be moved away from the observer, making the angles subtended by the front and rear shrink from $3.6^{\circ}$ and $3.8^{\circ}$ to the limits of acuity, and the proportions projected by the front and rear will always fall within the acceptable range. The picture, as it shrinks in subtense, can never project proportions that lie outside the range $0.95 \pm 0.12$.

The vantage point geometry of cubes and the criteria for acceptability offer a basis for perspective robustness. At large angular subtenses, a small change in distance produces large angular changes and, importantly, large changes in proportion too. The rate of change of the angle $y$ subtended by the front face, for any given angle $x$, subtended by the rear face, is $d y / d x=1+\cot ^{2} x /$ $\left(1+\cot ^{2} x\right)+4(1-\cot x)$. Cot $x$ is zero for large angles, leaving $d y / d x=1 / 5$. At small angles, $d y / d x=1$. Conversely, at small angular subtenses, a considerable change in distance produces small changes in angle, and, we stress, the changes in proportion that result are often quite modest. A change from $1.4^{\circ}$ (Hagen's optical infinity) to 
$5.7^{\circ}$ is a change of $30 \mathrm{w}$. The change in angular subtense is $4.3^{\circ}$ and the change in proportion is 0.1 . The physical principle here is that the proportions vary little for a movement of several times the width of the cube, when the drawing subtends a small angle (say $7^{\circ}$, give or take $2^{\circ}$ perhaps). The central psychological principle is sensitivity to correct projection, at a given angular subtense, allied to a range of acceptable variation. The principle of acceptable variation can explain fragile and robust perspective as follows.

Assume that a parallel projection drawing of a cube is deemed to be correct by the observer at small angular subtenses-at $0^{\circ}$, with the front-face to rear-face proportion being 1.0. Next, assume that the observer has a tolerance for variation. If the tolerance is 0.05 , the cube drawing is acceptable until the front face is $3.8^{\circ}$. If the tolerance is 0.08 , the drawing can have a front face of $4.8^{\circ}$. For an observer $\mathrm{O}_{17}$ with 0.17 tolerance, the front face can subtend $11.4^{\circ}$.

For $\mathrm{O}_{17}$, a polar projection drawing with the front face $9.5^{\circ}$ and the rear face $8.2^{\circ}(0.86)$ is acceptable at any angular subtense down to $0^{\circ}$. A cube with front face subtense of $14^{\circ}$ has proportions of 0.81 , just beyond the 0.17 cutoff for the parallel projection drawing. Furthermore, a cube with front face $19^{\circ}$ has 0.74 proportions. This proportion of 0.74 is within the 0.17 tolerance range of a 9.5/8.2 drawing with proportion 0.86 . Only when the front subtends $28^{\circ}$, where the correct proportion is 0.68 , is the 0.86 drawing beyond the 0.17 tolerance range-for the 0.68 is 0.01 beyond $0.86 \pm 0.17$. The result is that the $9.5^{\circ}$ front face drawing is acceptable from $0^{\circ}$ to just before $28^{\circ}$ for $\mathrm{O}_{17}$, using a 0.17 tolerance range.

Conversely, a drawing that the observer finds to be correct at $28^{\circ}$ cannot move by a distance of $4 w$ before it steps beyond the tolerance range of $\mathrm{O}_{17}$. Evidently a $28^{\circ}$ frontface cube would be extremely fragile at 0.17 tolerance, in comparison with a $9.5^{\circ}$ front-face cube, which would be acceptable from just below $28^{\circ}$ to $0^{\circ}$. This is all the range covered by the parallel drawing, and considerably more. Also it includes most of the distance covered by the $28^{\circ}$ cube $(4 \mathrm{w}$ being a distance that reduces the front face from a subtense of $28^{\circ}$ to $9.5^{\circ}$ ) plus the enormous distance that is necessary to reduce $9.5^{\circ}$ to close to $0^{\circ}$.

Notice that a $10 \times 10 \mathrm{~cm}$ photograph of a cube would subtend $9.5^{\circ}$ at $60 \mathrm{~cm}$, an arm's length. It would not move beyond, say, a 0.1 criterion range if it was brought to $40 \mathrm{~cm}$ or moved to $130 \mathrm{~cm}$. As a result, the everyday experience of holding photographs and looking at them from a wide range of distances, and finding them acceptable, fits quite nicely with the proposal that vision uses a proportion, wedded to a subtense, and a range of acceptable variation.

An interesting rider to this analysis is the issue of symmetry. It may be that some tolerance ranges are symmetrical. That is, if 0.80 is correct, the observer may tolerate variation of 0.1 on either side, accepting 0.7 as readily as 0.9 . Alternatively, tolerance in one direction may be much more acceptable. Notice that if 0.95 is correct, a variation of 0.1 would involve 0.85 (a polar projection drawing showing convergence with distance) and 1.05 (a drawing showing divergence with distance). It seems likely that tolerance ranges are pruned severely when they fall into divergence. At the opposite extreme, if the rear face of the cube is drawn as $53^{\circ}$, the proportion of that face to the front face cannot go beyond 0.29 , or the front face would subtend more than $180^{\circ}$. Hence, an interesting issue is the relationship between constraints on symmetry and the tolerance level selected by the observer. We commend this issue to further investigations.

Although we have good evidence that changing the angular subtense along a perpendicular to the vantage point strongly affects the choice of appropriate proportions, and variation in acceptable proportions could produce both apparent robustness and fragility, there remains one issue for which we confess we have no fully satisfactory explanation. It is not clear to us why some drawings of cubes are rated higher at their correct subtenses than others are. The $15^{\circ}$ cube in our experiments collected the best rating of all. It may be that subjects have in mind some prototype or best vantage point, in addition to projections that are simply correct.

This account of proportions and angles subtended has three valuable outcomes. It explains perceptual changes in the face of perspective invariants. It also predicts the perceptual changes correctly. It avoids problems incurred by a theory of limits to the visual field. Let us consider these points in order.

First, perceptual change occurs despite the presence of the cross-ratio, a perspective invariant (Cutting, 1986; Gibson, 1979). The cross-ratio remains constant no matter what the angular subtense of the object is. It is invariant in both natural and artificial perspective, as Cutting emphasized. Hence, it cannot explain changes in ratings when subtense changes.

Second, when the strongly convergent drawing is examined at a small angular subtense, it appears to be too sharply pointed to be a cube. Its proportions are far below the approximately 1.00 required for a tiny subtense. Hence, it should look as though it is too large in the front and too small in the rear. Indeed, inspection reveals that its base appears to shelve upward to the rear, and its rear edges seem too short to form a cube, yielding an impression of acute angles at the front. Quite the reverse, the parallel drawing looks too blunt when viewed at a large subtense, as inspection reveals. Its proportion is 1.00 , far above what is required for a cube at a large subtense. Hence, its front is too small, and its back is too large. It appears to flare from the front, diverging with distance, and its front angles appear obtuse when viewed with a large subtense.

Third, Kubovy (1986, chap. 7) links apparent distortion in viewing drawings of cubes subtending larger than $30^{\circ}$ to visual field limits. In Experiment 2, differences were obtained between the moderately convergent drawing and the parallel projection, both of which fit well within the $30^{\circ}$ limits described by Kubovy.

Finally, a comment on method: We have tried variations on this experiment in which subjects have been asked 
to estimate angles and lengths of the sides of the objects depicted by these displays, or to set the displays at the distances where they appear to be most like cubes-that is, to have right-angled corners and equal sides. These experiments produced considerable variability in response. The variability was particularly dramatic with the strongly convergent drawing (Nicholls \& Kennedy, 1992). Some subjects, we concluded after debriefing interviews, know that perspective produces unusual drawings, and so they deem drawings showing strong perspective effects to be quite acceptable renderings of cubes. Also, this reasoning leads them to place the strongly convergent drawing at a location where it "shows the perspective," one might say, rather than "shows the cube." We have found that our rating method does not raise the same difficulties.

\section{REFERENCES}

ARnheIM, R. (1974). Art and visual perception (2nd ed.). Berkeley \& Los Angeles: University of California Press.

Arnheim, R. (1977). Perception of perspective pictorial space from different viewing points. Leonardo, 10, 283-288.

Bengston, J. K., Stergios, J. C., Ward, J. L., \& Jester, R. E. (1980). Optic array determinants of apparent distance and size in pictures. Journal of Experimental Psychology: Human Perception \& Performance, 6, 751-759.

Busey, T. A., Brady, N. P., \& Cutting, J. E. (1990). Compensation is unnecessary for the perception of faces in slanted pictures. Perception \& Psychophysics, 48, 1-11.

Cutting, J. E. (1986). Perception with an eye for motion. Cambridge, MA: MIT Press.

Cutting, J. E. (1987). Rigidity in cinema seen from the front row, side aisle. Journal of Experimental Psychology: Human Perception \& Performance, 13, 323-334.

Dubery, F., \& Willats, J. (1983). Perspective and other drawing systems. London: Herbert Press.

EDGERTON, S. Y. (1975). The Renaissance rediscovery of linear perspective. New York: Basic Books.

FARBER, J., \& RoSINSKI, R.R. (1978). Geometric transformations of pictured space. Perception, 7, 269-282.

GiBson, J. (1979). The ecological approach to visual perception. Boston: Houghton-Mifflin.

GoLDSTEIN, E. B. (1987). Spatial layout, orientation relative to the observer, and perceived projection in pictures viewed at an angle. Journal of Experimental Psychology: Human Perception \& Performance, 13, 256-266.

Hagen, M. A. (1980). Generative theory: A perceptual theory of pictorial representation. In M. A. Hagen, (Ed.), The perception of pictures (Vol. 2, pp. 3-46). New York: Academic Press.

HaGeN, M.A. (1985). There is no development in art. In N. H. Freeman \& M. V. Cox (Eds.), Visual order (pF. 59-77). Cambridge: Cambridge University Press.

Hagen, M. A. (1986), Varieties of realism. Cambridge: Cambridge University Press.

Kubovy, M. (1986). The psychology of perspective and Renaissance art. Cambridge: Cambridge University Press.

Lumsden, E. A. (1980). Problems of magnification and minification: An explanation of the distortions of distance, slant, shape and velocity. In M. A. Hagen (Ed.), The perception of pictures (Vol. 1, pp. 91135). New York: Academic Press.

MCGreevy, M. W., \& Ellis, S. R. (1986). The effect of perspective geometry on judged direction in spatial information instruments. $\mathrm{Hu}$ man Factors, 28, 439-456.

Nicholls, A. L., \& Kennedy, J. M. (1992, June). Perspective robustness and optical infinity. Paper presented at the meeting of the Canadian Society for Brain, Behavioural and Cognitive Sciences, Quebec City.

Pirenne, M. H. (1970). Optics, painting and photography. Cambridge: Cambridge University Press

RosinsKI, R. R., \& FARBER, J. (1980). Compensation for viewing point in the perception of pictured space. In M. A. Hagen (Ed.), The perception of pictures (Vol. 1, pp. 137-176). New York: Academic Press.

Rosinski, R. R., Mulholland, T., Degelman, D., \& Farber, J. (1980). Picture perception: An analysis of visual compensation. Perception \& Psychophysics, 28, 521-526.

SEDGWICK, H. A. (1980). The geometry of spatial layout in pictorial representation. In M. A. Hagen (Ed.), The perception of pictures (Vol. 1, pp. 33-90). New York: Academic Press.

SEDGWICK, H. A. (1991). The effects of viewpoint on the virtual space of pictures. In S. R. Ellis (Ed.), Pictorial communication in virtual and real environments (pp. 460-479). New York: Taylor \& Francis.

SMrTH, O.W. (1958). Judgments of size and distance in photographs. American Journal of Psychology, 71, 529-538.

Smith, O. W., \& Gruber, H. (1958). Perception of depth in photographs. Perceptual \& Motor Skills, 8, 307-313.

Veltman, K. H. (1987). Studies on Leonardo da Vinci I: Linear perspective and the visual dimensions of art and science. Munich: Deutscher Kunstverlag.

Wallach, H., \& Marshall, F. J. (1986). Shape constancy in pictorial representation. Perception \& Psychophysics, 39, 233-235.

WhITE, J. (1967). The birth and rebirth of pictorial space. Somerset, U.K.: Butler \& Tanner.

\section{APPENDIX A}

Angular Subtenses in Degrees of Three Drawings of a Cube for Five Viewing Distances in Experiments 1 and 2

\begin{tabular}{lrrrrr}
\hline Drawing & $35^{\circ}$ & $15^{\circ}$ & $5^{\circ}$ & $4^{\circ}$ & $2^{\circ}$ \\
$\quad 20 \mathrm{~cm}$ & $48 \mathrm{~cm}$ & $140 \mathrm{~cm}$ & $200 \mathrm{~cm}$ & $400 \mathrm{~cm}$ \\
\hline $\begin{array}{l}\text { Parallel } \\
\text { Height }\end{array}$ & 37.8 & 16.2 & 5.6 & 3.9 & 1.9 \\
$\quad$ Width & 35.2 & 15.1 & 5.2 & 3.6 & 1.8 \\
Moderately Convergent & & & & & \\
$\quad$ Height & 34.2 & 14.6 & 5.0 & 3.5 & 1.8 \\
$\quad$ Width & 34.2 & 14.6 & 5.0 & 3.5 & 1.8 \\
Strongly Convergent & & & & & \\
$\quad$ Height & 32.9 & 14.0 & 4.8 & 3.4 & 2.0 \\
$\quad$ Width & 36.0 & 15.4 & 5.2 & 3.7 & 1.9 \\
\hline
\end{tabular}


APPENDIX B

Angular Subtenses in Degrees of Three Drawings of a Cube for Five Viewing Distances in Experiment 3

\begin{tabular}{llllll}
\multicolumn{1}{c}{ Drawing } & $\begin{array}{c}50^{\circ} \\
13 \mathrm{~cm}\end{array}$ & $\begin{array}{c}45^{\circ} \\
15 \mathrm{~cm}\end{array}$ & $\begin{array}{c}40^{\circ} \\
17 \mathrm{~cm}\end{array}$ & $\begin{array}{c}35^{\circ} \\
20 \mathrm{~cm}\end{array}$ & $\begin{array}{c}30^{\circ} \\
23 \mathrm{~cm}\end{array}$ \\
\hline Parallel & & & & & \\
$\quad$ Height & 55.6 & 49.1 & 44.0 & 37.8 & 33.2 \\
$\quad$ Width & 52.07 & 45.89 & 40.96 & 35.2 & 30.9 \\
$\quad \begin{array}{l}\text { Moderately Convergent } \\
\quad \text { Height }\end{array}$ & 50.6 & 44.6 & 39.8 & 34.2 & 29.9 \\
$\quad$ Width & 50.6 & 44.6 & 39.8 & 34.2 & 29.9 \\
$\begin{array}{l}\text { Strongly Convergent } \\
\quad \text { Height }\end{array}$ & & & & & \\
$\quad$ Width & 48.8 & 42.9 & 38.3 & 32.9 & 28.8 \\
\hline
\end{tabular}

(Manuscript received July 16, 1992;

revision accepted for publication May 28, 1993.) 